\title{
Recouvrement des pertes de substance de la pulpe des doigts par des lambeaux locaux à Hanoï, Vietnam
}

\section{Covering of Finger-Tissue Loss by Local Flaps in Hanoi, Vietnam}

\section{D.T. Nguyen - T. S. Tran · B. H. Nguyen - V. D. Pham}

Reçu le 31 janvier 2018; accepté le 1 mai 2018

(C) Société de pathologie exotique et Lavoisier SAS 2018

Résumé Une étude sur les pertes de substance accidentelles de la pulpe des doigts et leur recouvrement par lambeaux locaux a été réalisée dans le service de chirurgie reconstructive de l'hôpital Saint Paul de Hanoï de janvier 2016 à juin 2017. Elle a concerné 30 patients, 24 hommes et 6 femmes. La cause la plus fréquente était l'accident de travail, soit 21/30 cas. Au moment de l'accident, 12 patients ne disposaient pas d'équipement de protection individuelle (EPI). Sur les 18 patients qui en possédaient, 8 avaient un équipement incomplet. Sur 32 lambeaux mis en place, 31 ont survécu complètement sans nécrose, ni infection, et un a subi une épidermolyse. Trente et un des 32 doigts opérés ont conservé une fonction motrice de bonne qualité et 27 ont récupéré une sensibilité de niveau $\mathrm{S} 4$. Le traitement en urgence des pertes de substance de la pulpe des doigts par des lambeaux locaux permet de préserver la fonction motrice fine et la sensibilité des pulpes des doigts.

Mots clés Pulpe des doigts - Perte de substance · Lambeaux locaux $\cdot$ Chirurgie réparatrice $\cdot$ Accident du travail · Hôpital · Hanoï · Vietnam · Asie du Sud-Est

\begin{abstract}
Abtract The accidental loss of fingertip soft tissues, which may expose tendons and bones, is a common injury in emergency departments. If these lesions are poorly treated, they can impair fine motor skills and tactile sensitivity of the fingertips. The study was conducted on 30 patients ( 24 males and 6 females) with 32 soft tissue defects of the fingertip treated in emergency plastic surgery with local pedicled flap at the Plastic Surgery Department of Saint Paul Hospital
\end{abstract}

D.T. Nguyen $(\bowtie) \cdot$ T. S. Tran · B. H. Nguyen · V. D. Pham Department of surgery in Hai Phong University of Medicine and Pharmacy, 72A Nguyen Binh Khiem,

Ngo Quyen (district), Hai Phong, Vietnam

e-mail : nguyenductiendhy@gmail.com

Communication présentée au $\mathrm{X}^{\mathrm{e}}$ Congrès international de la Société de pathologie exotique à Haïphong, 9-10 novembre 2017
Hanoi from $01 / 2016$ to $06 / 2017$. The most common cause of injury (21/30) was occupational accidents. At the time of the accident, 12 patients did not have personal protective equipment (PPE). Among 18 patients who had one, eight had incomplete equipment. Of 32 implanted skin flaps, 31 survived completely without necrosis or infection, only one being affected by epidermolysis. Postoperative evaluation showed excellent motor skills for 31/32 fingers and a sensitivity restoration at S4 level for 27/32. Workplace accident is the main cause of fingers soft tissue defects. Covering the fingers soft tissue defects with local pedicled flap in emergency preserves the fine motor function and the delicated tactile sensation of the fingers.

Keywords Fingertip - Soft tissue defect - Local flap · Restorative surgery · Occupational accident $\cdot$ Hospital Hanoi · Vietnam $\cdot$ South East Asia

\section{Introduction}

Les plaies de l'extrémité distale des doigts sont fréquemment observées après des accidents du travail, des accidents domestiques et des accidents de la circulation. Elles concernent fréquemment la pulpe des doigts et requièrent une prise en charge en urgence. Le recouvrement des pertes de substance doit respecter cinq principes :

- préserver la longueur maximale des doigts ;

- conserver la couche de graisse sous-cutanée recouvrant la plaie ;

- restaurer la sensibilité et la motricité fines de la pulpe des doigts ;

- fournir suffisamment de cellules stromales pour permettre la croissance de l'ongle ;

- être indolore, permettant au patient de reprendre rapidement ses activités quotidiennes et laisser un minimum de séquelles au niveau du site de prélèvement. 
Notre étude, menée dans le service de chirurgie reconstructrice de l'hôpital Saint Paul de Hanoi, de janvier 2016 à juin 2017, avait pour objectifs de décrire les caractéristiques cliniques et d'évaluer les résultats des pertes de substance de la pulpe des doigts recouvertes en urgence par des lambeaux cutanés.

\section{Population d'étude et méthodologie}

Il s'agit d'une étude descriptive longitudinale portant sur les dossiers médicaux de 30 patients ayant subi 32 pertes de substance de la pulpe des doigts, dues à différentes causes, recouvertes par des lambeaux cutanés locaux,

\section{Critères d'inclusion}

Tous les patients présentaient des pertes de substance de la pulpe des doigts avec exposition tendineuse et osseuse. Ces patients ont été examinés, ont bénéficié d'examens paracliniques (numération et formule sanguine, bilan de coagulation et radiographie de la main en position droite et inclinée). Ces patients ont été opérés, traités médicalement et revus en consultation une semaine, un mois, trois mois et six mois après la sortie de l'hôpital.

\section{Critères d'exclusion}

- toute atteinte de l'ensemble du périmètre de la pulpe des doigts nécessitant une couverture par des lambeaux provenant d'autres localisations ;

- les polytraumatismes avec menace vitale nécessitant en priorité d'autres interventions chirurgicales, l'opération de reconstruction de la pulpe digitale n'étant envisagée qu'une fois l'état général stabilisé ;

- les cas ayant été traités par d'autres méthodes : couverture par greffe de peau, réimplantation de pulpe ou cicatrisation dirigée, etc. ;

- les plaies infectées de la pulpe des doigts.

\section{Variables de l'étude}

Pour chaque patient, les caractéristiques suivantes ont été colligées : sexe, âge, utilisation d'équipements de protection individuelle, causes, lieu et type de blessure, type de lambeau utilisé et capacité de survie de chaque type de lambeau, temps de cicatrisation, résultat post-opératoire à 6 mois, état des cicatrices des zones donneuses de lambeaux.

Le rétablissement de la sensibilité a été évalué selon la classificationde de McKinnon-Dellon qui distingue 5 niveaux à l'aide de deux indicateurs : discrimination de 2 points statiques (s2PD) et de 2 points dynamiques
(m2PD). Selon cette classification, il n'y a aucune restauration de la sensibilité au niveau $\mathrm{S} 0$, une faible restauration aux niveaux S1 à S2+, une restauration de la douleur et du toucher au niveau $\mathrm{S} 3$ et une restauration complète de la sensibilité au niveau S4.

La récupération de la fonction motrice a été mesurée à l'aide de la classification de l'Association américaine pour la chirurgie du traumatisme (AAST) qui distingue 5 niveaux :

- bon : amplitude du mouvement articulaire restaurée dans la limite de la normale ;

- assez bon : amplitude du mouvement articulaire supérieure à $75 \%$ de la valeur normale ;

- moyen : amplitude du mouvement articulaire entre 50 et $75 \%$ de la valeur normale ;

- médiocre : amplitude du mouvement articulaire inférieure à $50 \%$ de la valeur normale ;

- pas de mouvement : raideur articulaire, aucun mouvement articulaire.

\section{Résultats}

De janvier 2016 à juin 2017, 30 patients, 24 hommes et 6 femmes âgés de 2 à 70 ans, ont été opérés par les lambeaux locaux pour 32 pertes de substance de la pulpe des doigts. Six travaillaient dans le secteur industriel du cuir et de la chaussure, 5 étaient ouvriers dans des entreprises spécialisées dans la fabrication de pièces de machines industrielles, 5 étaient bouchers et 6 menuisiers.

La cause la plus fréquente ( $21 \mathrm{cas}$ ) était l'accident de travail. Parmi ces derniers, 8 patients hospitalisés n'avaient pas bénéficié de premiers secours sur le lieu de l'accident. Parmi les 13 autres, 5 avaient reçu des premiers soins inappropriés : immersion de la partie libre du doigt dans l'eau glacée pour 3 patients et 2 garrots du poignet au lieu d'un pansement compressif (Figs 1, 2).

Concernant le traitement, la taille du plus grand lambeau était de $3,5 \times 1,5 \mathrm{~cm}$, celle du plus petit lambeau de $1 \times 1$, $5 \mathrm{~cm}$. Nous avons utilisé de manière flexible deux types de lambeau : lambeau libre et lambeau en îlot. Des lambeaux sont prélevés au voisinage de la lésion et mis en forme en fonction de la perte de substance.

Le coût moyen du traitement était de 10 millions de VND (environ 500 \$ US), dont $30 \%$ pour les actes chirurgicaux et $70 \%$ pour les médicaments, les examens paracliniques et les frais d'hospitalisation. Sur 30 patients, $16(53 \%)$ ont été remboursés à $80 \%$ de leurs frais de traitement par l'assurance maladie, $6(20 \%)$ ont bénéficié d'une prise en charge de la totalité des frais de traitement par leur entreprise et 8 ( $27 \%$ ) ont dû assumer la totalité du coût de leur traitement. 

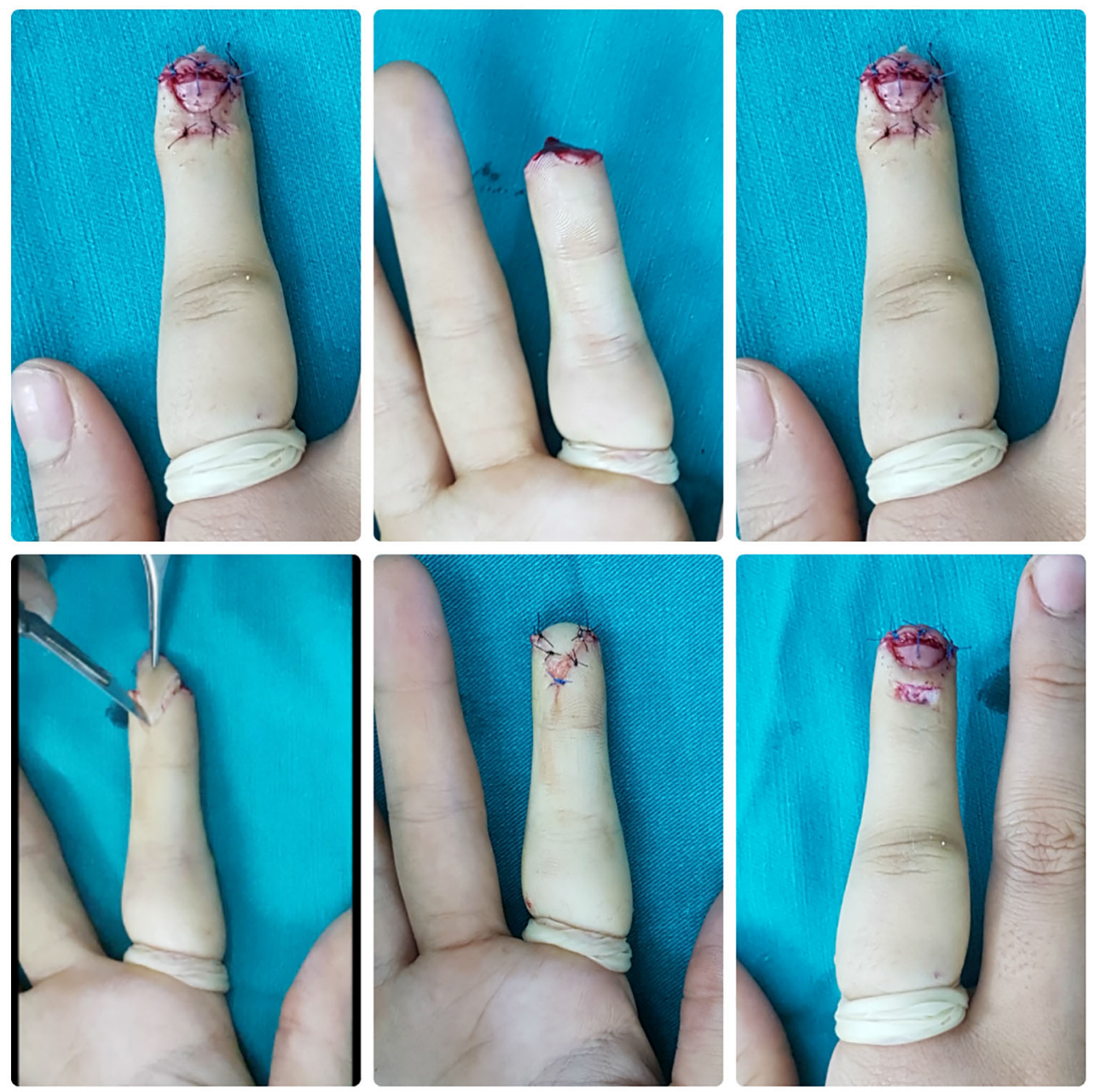

Fig 1 Patient de 27 ans, travailleur manuel, victime d'un écrasement pulpaire du $2^{\mathrm{e}}$ doigt de la main droite, au niveau de la zone 2 selon la classification d'Ailen / 27-year old patient, manual worker, victim of a fingertip crush of the $2^{\text {nd }}$ finger of the right hand, at Zone 2 according to Ailen classification

Il n'a pas été constaté de nécrose ou d'infection au niveau des lambeaux. L'évaluation clinique post-opératoire effectuée à 6 mois n'a révélé aucune cicatrice hypertrophique, ni chéloïde. Elle a montré que 31/32 lambeaux avaient restitué une fonction motrice des doigts de bon niveau et que 27/32 avaient permis de restaurer une sensibilité de niveau $\mathrm{S} 4$, les lambeaux libres permettant une meilleure restauration de la sensibilité que les lambeaux en îlot.

\section{Discussion}

$\mathrm{Au}$ Vietnam, la chirurgie reconstructrice de la main n'est actuellement développée que dans les grands hôpitaux de Hanoi et Ho Chi Minh ville. Ailleurs, les blessés de la main sont pris en charge dans les services de chirurgie générale, avec des traitements parfois peu efficaces. Pourtant, les acci- dents entraînant des pertes de substance de la pulpe des doigts sont fréquents [5]. Ce sont souvent des accidents du travail liés au fait que l'automatisation est encore très limitée dans les ateliers de production, ce qui expose la main au contact direct des outils [4]. De plus, les travailleurs et les employeurs sont encore peu sensibilisés à la prévention et aux mesures de protection au travail, bien qu'il existe des équipements de protection individuelle efficaces pour réduire le risque et la gravité des accidents. Par ailleurs, il existe peu de dispositifs de premiers secours et ceux-ci sont souvent de mauvaise qualité. Cette situation reflète le faible développement de la médecine du travail au Vietnam et son manque d'attractivité dans le cursus des formations médicales. De fait, la plupart des entreprises et des usines n'ont pas de service de médecine du travail. En outre, les personnels médicaux en entreprise ne sont pas formés correctement à la prise en charge initiale et aux premiers secours en cas d'accident [3]. 

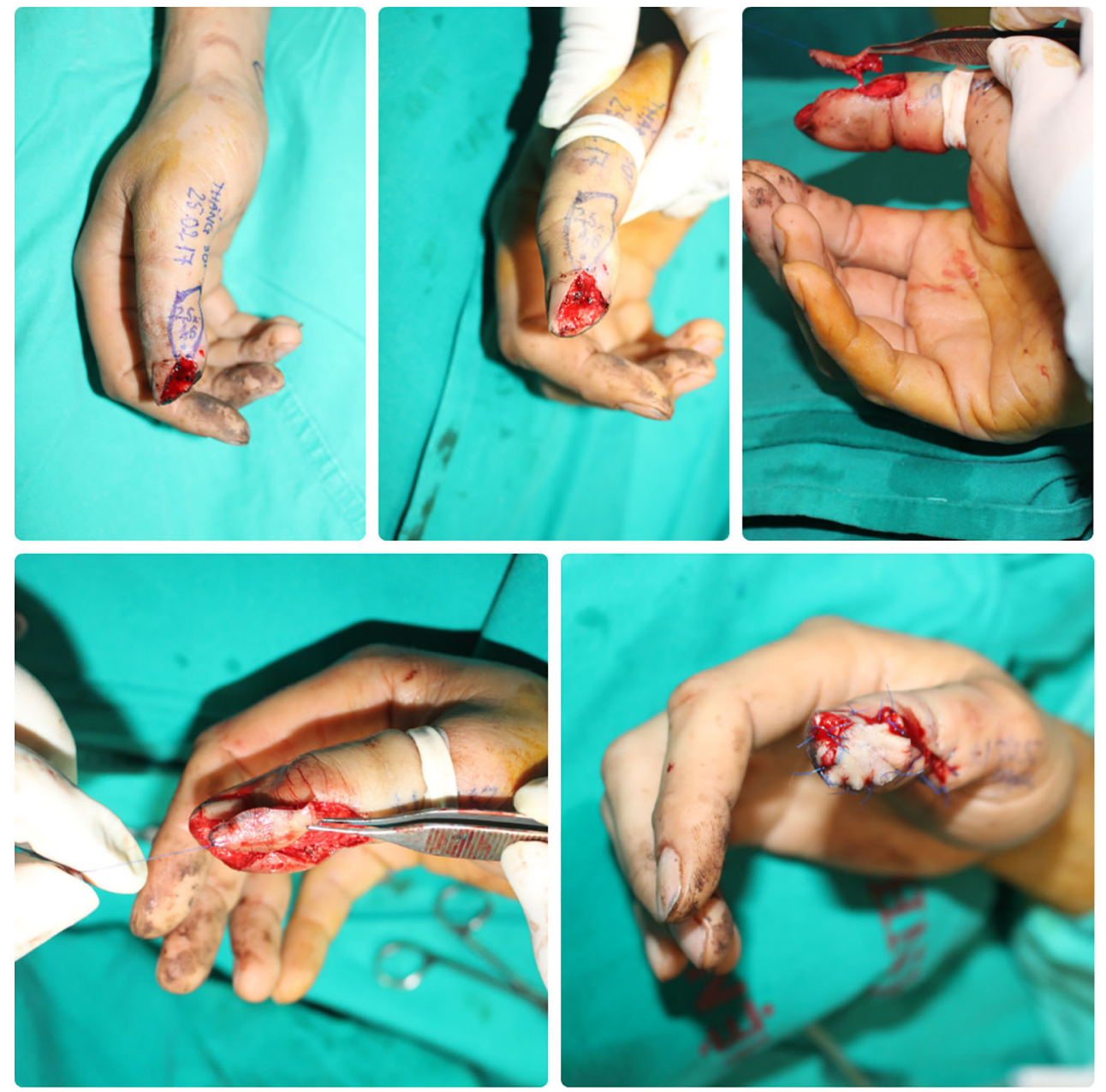

Fig 2 Patient de 18 ans, victime d'un accident du travail. Perte de substance à la marge radiale de la pulpe du $1^{\mathrm{er}}$ doigt de la main droite $(2,5 \mathrm{x} 1,5 \mathrm{~cm}) /$ 18-year old patient, victim of an occupational accident. Loss of fingertip soft tissues at the radial margin of the $1^{\text {st }}$ finger of the right hand

On peut recourir à plusieurs types de lambeaux pour recouvrir des pertes de substance de la pulpe des doigts, en fonction des caractéristiques morphologiques de la lésion. Dans notre pratique, nous avons utilisé des lambeaux en îlot ou des lambeaux libres. Les lambeaux libres ont l'avantage de la simplicité, mais ils recouvrent moins bien que les lambeaux en îlot [5]. Les pertes de substance de moins de $1 \mathrm{~cm}$, horizontales ou obliques au niveau de la face dorsale de la pulpe, ont été recouvertes par des lambeaux locaux. Pour les pertes de substance obliques palmaires et les pertes de substance latérales, nous avons choisi les lambeaux en îlot pour leur meilleure capacité de recouvrement [6].

L'évaluation post-opératoire de la fonction motrice montre que $31 / 32$ des doigts opérés présentent une amplitude satisfaisante dans le mouvement des articulations interphalangiennes distales, conformément aux séries publiées $[1,2]$.

\section{Conclusion}

Les accidents du travail sont la cause principale des pertes de substance de la pulpe des doigts au Vietnam. La prévention impose d'améliorer la prise de conscience des travailleurs et des employeurs sur l'intérêt d'utiliser des équipements de protection individuelle et de former les personnels médicaux des établissements de production aux premiers gestes de prise en charge. De plus, il est nécessaire d'ouvrir un service spécialisé de chirurgie réparatrice de la main dans chaque hôpital régional. L'utilisation des lambeaux locaux est la technique plastique qui conserve au mieux la morphologie ainsi que la fonction motrice fine et la sensibilité de la pulpe des doigts.

Liens d'intérêt : les auteurs déclarent ne pas avoir de liens d'intérêt. 


\section{Bibliographie}

1. Aboulwafa A, Emara S (2013) Versatility of Homodigital Islandized Lateral V-Y Flap for Reconstruction of Fingertips and Amputation Stumps. Egypt J Plast Reconstr Surg 37:89-96

2. Atasoy E, Ioakimidis E, Kasdan ML, et al (1970) Reconstruction of the amputated finger tip with a triangular volar flap. A new surgical procedure. J Bone Joint Surg Am. 52:921-6

3. Doan LV, al (2012) Revue Medicale Pratique No 6 68-71.
4. Foo TL, Wan KH (2012) Modification of VY flap to preserve fingertip contour. Hand. 7:388-90. doi: 10.1007/s11552-0129451-z.

5. Hoang NV, Son TT (2007) Tình hình phẫu thuật tạo hình vết thương bàn tay tại Bệnh viện Xanh Pôn, Revue Médicale du Vietnam. 339:99-07

6. Robert N, Chassat R, Couturier C, et al (2015) "Reposition-flap": A therapeutic alternative in fingertips amputations. Ann Chir Plast Esthet, 60:299-304 